\title{
High Li-ion Conductivity Artificial Interface Enabled by Li-grafted Graphene Oxide for Stable Li Metal Pouch Cell
}

Jianhua Liang ${ }^{a, b, \dagger}$, Wei Deng ${ }^{b,}+$, Xufeng Zhou ${ }^{*}, b$ Shanshan Liang ${ }^{b}$, Zhiyuan $\mathrm{Hu}^{b}$, Bangyi He ${ }^{b}$, Guangjie Shao ${ }^{*}$, , and Zhaoping Liu* ${ }^{*}, b$

a State Key Laboratory of Metastable Materials Science and Technology, College of Environmental and Chemical Engineering, Yanshan University, Qinhuangdao 066004, China

b Advanced Li-ion Battery Engineering Laboratory of Zhejiang Province, Key Laboratory of Graphene Technologies and Applications of Zhejiang Province, and CAS Engineering Laboratory for Graphene, Ningbo Institute of Materials Technology \& Engineering, Chinese Academy of Sciences, Zhejiang 315201, P. R. China

$\dagger$ These authors contribute equally to this work.

* Corresponding authors

E-mail address: zhouxf@nimte.ac.cn, shaogj@ysu.edu.cn, liuzp@nimte.ac.cn 


\section{EXPERIMENTAL SECTION}

\section{Preparation of GO-ADP- $\mathrm{Li}_{3}$}

Graphene oxide (GO) was prepared according to the method and procedures previously reported by our group. ${ }^{1}$ Adenosine 5 '-diphosphate lithium (ADP-Li ${ }_{3}$ ) was purchased from RHAWN (purity of $98 \%$ ). The Li-grafted GO composites were synthesized by the reaction between GO and ADP-Li 3 . Firstly, $200 \mathrm{mg}$ of freeze-dried GO was added to a beaker containing $200 \mathrm{ml}$ of DMF, and the mixture was ultrasonicated for $0.5 \mathrm{~h}$. Then, the beaker was transferred to an ice bath with a mixture of ice and water at $0^{\circ} \mathrm{C}$. Subsequently, $9.2 \mathrm{~g}$ of 3-(ethyliminomethylideneamino)-N,N-dimethylpropan-1amine/hydrochloride (EDC/HCl) and $5.6 \mathrm{~g}$ of N-hydroxy-succinimide (NHS) were added to the beaker and stirring was continued for $1 \mathrm{~h}$. Subsequently, the beaker was taken out of the ice-water bath, and $1 \mathrm{~g}$ of GO-ADP- $\mathrm{Li}_{3}$ was added to the beaker and stirred at $25^{\circ} \mathrm{C}$ for $12 \mathrm{~h}$. The grafted product (GO-ADP-Li $\left.i_{3}\right)$ was filtered and washed with DMF several times. Finally, the GO-ADP- $\mathrm{Li}_{3}$ obtained by suction filtration was freeze-dried by lyophilizer.

\section{Fabrications of the SPE}

For the fabrication of the SPE, firstly, $\mathrm{x} \operatorname{mg}(\mathrm{x}=0,16,24,40,80,120$ and $160 \mathrm{mg})$ of GO or GO-ADP-Li ${ }_{3}$ and $500 \mathrm{mg}$ of lithium bis-trifluoromethanesulfonimide (LiTFSI) were added in a $20 \mathrm{~mL}$ glass bottle in the argon-filled glove box with oxygen and water contents blow $0.1 \mathrm{ppm}$. Then, $8.0 \mathrm{~mL}$ of 1-methyl-2-pyrrolidinone (NMP) was added. The glass bottle was sealed and subjected to stirring for $0.5 \mathrm{~h}$. After that, $1.0 \mathrm{~g}$ of poly(vinylidene-co-hexafluoropropylene) (PVDF-HFP) was added to the mixture and 
stirred for $24 \mathrm{~h}$ at room temperature. The mixture was scraped onto the glass plate by film casting blade and transferred to the vacuum oven under $70{ }^{\circ} \mathrm{C}$ for $6 \mathrm{~h}$. The SPE was cut into dics with a diameter of $19.0 \mathrm{~mm}$. For the fabrication of Li-GO-SPE on $\mathrm{Cu}$ foil or Li metal, firstly, the GO-ADP-Li $i_{3} / \mathrm{LiTFSI} / \mathrm{PVDF}-\mathrm{HFP} / \mathrm{NMP}$ slurry was scraped onto the $\mathrm{Cu}$ foil or $\mathrm{Li}$ foil $(100 \mu \mathrm{m})$ in the drying room with dew point of $-60{ }^{\circ} \mathrm{C}$. Then the composites were transferred to the vacuum oven and followed by drying at $70{ }^{\circ} \mathrm{C}$ for 6 h. The Li-GO-SPE@Cu or Li-GO-SPE@Li was punched to form discs with a diameter of $14.0 \mathrm{~mm}$ for coin cells and was cut into size of $57 \mathrm{~mm} \times 47 \mathrm{~mm}$ for pouch cells.

3 Material characterizations

Morphology of GO/GOADP- $\mathrm{Li}_{3}$ and all pristine and cycled electrodes were characterized by the Hitachi S-4800 field emission scanning electron microscope (SEM), the Tecnai F20 transmission electron microscope (TEM) and the Helios-G4CX focused ion beam (FIB). The X-ray photoelectron spectroscopy (XPS) was detected by a Kratos Axis Ultra DLD and the FT-IR spectra were recorded using Nicolet iS50 spectrophotometer ( $\mathrm{KBr}$ tablets). The glass transition temperature was examined on differential scanning calorimetry (DSC 214). The thermogravimetric analysis curves was examined on thermogravimetric differential thermal integrated thermal analyzer (TG/DTA).

\section{Electrochemical measurement}

Standard CR2032 coin-type cell was employed for the assembly of $\mathrm{Li} / \mathrm{Cu}$ cells and Li symmetric cells. The assembly process was performed in an Ar-filled glove box, and 
both the oxygen and water content are blow $0.1 \mathrm{ppm}$. In all half-cells, $60 \mu \mathrm{L}$ of etherbased electrolyte composed by 1.0 M LiTFSI-DOL/DME (volume ratio of 1:1) with 1.0 wt. $\% \mathrm{LiNO}_{3}$ was used. During the galvanostatic test (battery test system, C2001A, LAND, China) of $\mathrm{Li} / \mathrm{Cu}$ cells, the current density of $0.5 \mathrm{~mA} \mathrm{~cm}{ }^{-2}$ and areal capacity of $3.0 \mathrm{mAh} \mathrm{cm}^{-2}$ was applied with the cut-off voltage of $1.0 \mathrm{~V}$. And for Li symmetric cells, the current density of $0.5 \mathrm{~mA} \mathrm{~cm}-2$ and areal capacity of $3.0 \mathrm{mAh} \mathrm{cm} \mathrm{m}^{-2}$ was applied. The EIS measurement was conducted in the frequency range of $10^{6} \sim 10^{-2} \mathrm{~Hz}$ under amplitude of $10 \mathrm{mV}$ by Solartron 1470E electrochemical workstation (Solartron Analytical, UK). The ionic conductivity $(\sigma)$ of electrolyte was tested by a configuration of sandwiching SPE between two stainless steel sheets. The temperature for ionic conductivity measurement ranged from 25 to $80{ }^{\circ} \mathrm{C}$. The calculation for ionic conductivity follows the equation below:

$$
\sigma=\frac{L}{R_{b} \times S}
$$

where $L, R_{b}$, and $S$ stand for the membrane thickness of SPE, the ohmic resistance revealed by EIS, and the cross-sectional area of SS, respectively. The ionic conductivity results were related to a typical Vogel-Tamman-Fulcher (VFT) relationship under the temperature range of $25 \sim 80{ }^{\circ} \mathrm{C}$,

$$
\sigma=\mathrm{A} T^{-\frac{1}{2}} \exp \left(\frac{-E_{a}}{R\left(T-T_{0}\right)}\right)
$$

where $\sigma$ is the ionic conductivity, A is a constant that depends on the charge carrier density, $\mathrm{R}$ is the universal gas constant, $\mathrm{E}_{\mathrm{a}}$ is the activation energy, $\mathrm{T}_{0}$ equals to glass transition temperature $\left(\mathrm{T}_{\mathrm{g}}\right)$ and $\mathrm{T}$ is the ambient temperature. To study the Li-ion transference number $\left(t_{\mathrm{Li}}^{+}\right)$of SPE, a coin cell of "Li/SPE/Li" was constructed. 
Electrochemical impedance spectroscopy (EIS) was measured in the frequency range from $10^{6} \mathrm{~Hz}$ to $10^{-2} \mathrm{~Hz}$ using a Solartron $1470 \mathrm{E}$ electrochemical workstation (Solartron Analytical, UK). EIS analysis was performed before and after chronoamperometry (CA). During CA a polarization voltage of $10 \mathrm{mV}$ was applied for $6000 \mathrm{~s}$ until a steady state was reached. $\mathrm{t}_{\mathrm{Li}}{ }^{+}$was calculated using the following equation,

$t_{L i}+=\frac{I_{s} R_{b 0}\left(\Delta V-I_{0} R_{0}\right)}{I_{0} R_{b s}\left(\Delta V-I_{s} R_{s}\right)}$

where $\mathrm{t}_{\mathrm{Li}}{ }^{+}$is the Li-ion transference number, $\Delta \mathrm{V}$ is the constant voltage applied to the cell, $\mathrm{I}_{0}$ and $\mathrm{I}_{\mathrm{S}}$ stand for initial current and steady-state currents in the polarization process, respectively, $\mathrm{R}_{0}$ and $\mathrm{R}_{\mathrm{S}}$ represent the interfacial resistances of SPE before and after polarization, respectively, and $\mathrm{R}_{\mathrm{b} 0}$ and $\mathrm{R}_{\mathrm{bS}}$ represent the bulk impedance of SPE before and after polarization, respectively. For the assessment of electrochemical stability of Li-GO-SPE, a configuration of "Li/ Li-GO-SPE /SS (stainless steel)" was constructed. The linear sweep voltammetry (LSV) curves were obtained by using the Solartron 1470E electrochemical workstation (Solartron Analytical, UK) from 0 to 5.0 $\mathrm{V}$ at a scanning rate of $0.5 \mathrm{mV} \mathrm{s}^{-1}$.For pouch full cells, bare Li foil $(100 \mu \mathrm{m})$, GOSPE@Li foil (100 $\mu \mathrm{m})$ and Li-GO-SPE@Li foil $(100 \mu \mathrm{m})$ with the size of $57 \mathrm{~mm} \times 47$ mm were used as the anode, and the commercial NCM811 with size of $53 \mathrm{~mm} \times 43$ $\mathrm{mm}$ as the cathode. Commercial PP membrane ( $25 \mu \mathrm{m}$ in thickness) was employed as the separator. The injection ratio of electrolyte (1.0 $\mathrm{M} \mathrm{LiPF}_{6}$ in $\mathrm{EC} / \mathrm{DMC}$ (volume ratio of $1: 1$ ), with $2 w t . \%$ FEC as additive) is $3.0 \mathrm{~g} / \mathrm{Ah}$. The voltage window range for pouch cell is 2.6 to $4.3 \mathrm{~V}$. The pouch cells were cycled at $20 \mathrm{~mA}$ (corresponding to $0.1 \mathrm{C}$ ). Detailed fabrication process can be seen in our previous report. ${ }^{2}$ 
1. Supplementary figures

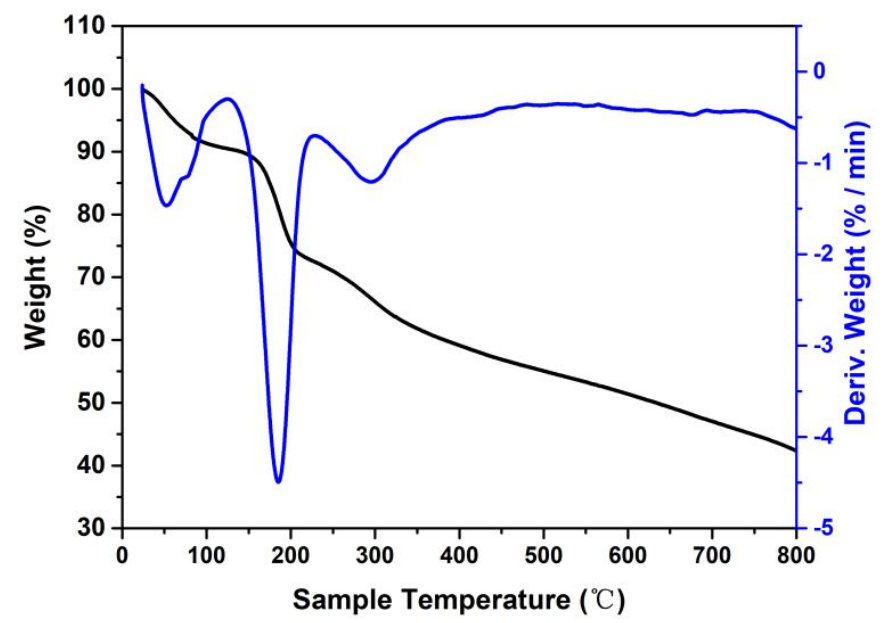

Figure S1. Thermogravimetric analysis curves (TGA) and the corresponding derivative thermogravimetric curves of GO-ADP- $\mathrm{Li}_{3}$.
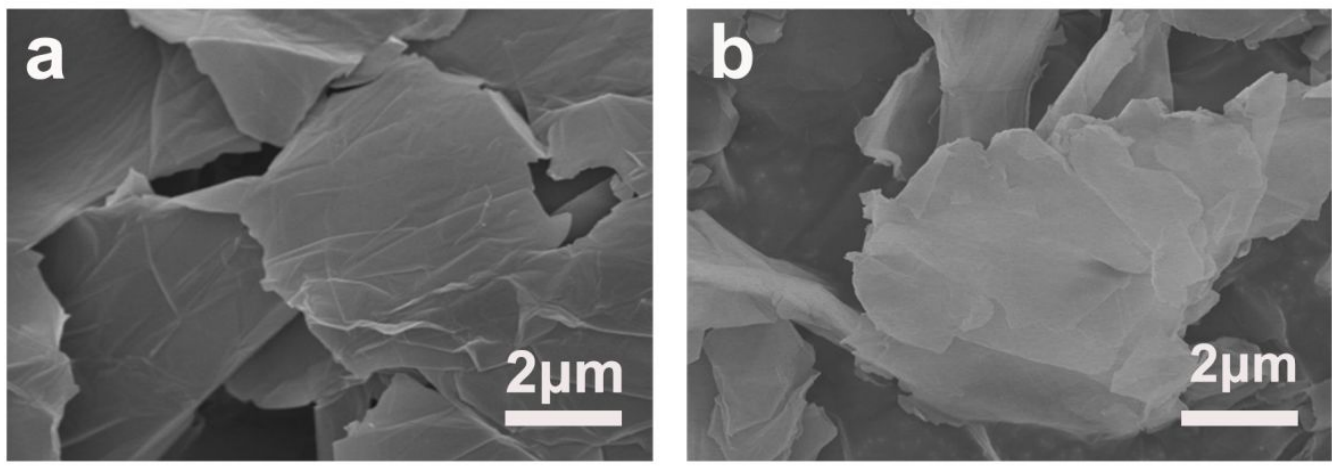

Figure S2. SEM images of (a) GO and (b) GO-ADP-Li. 

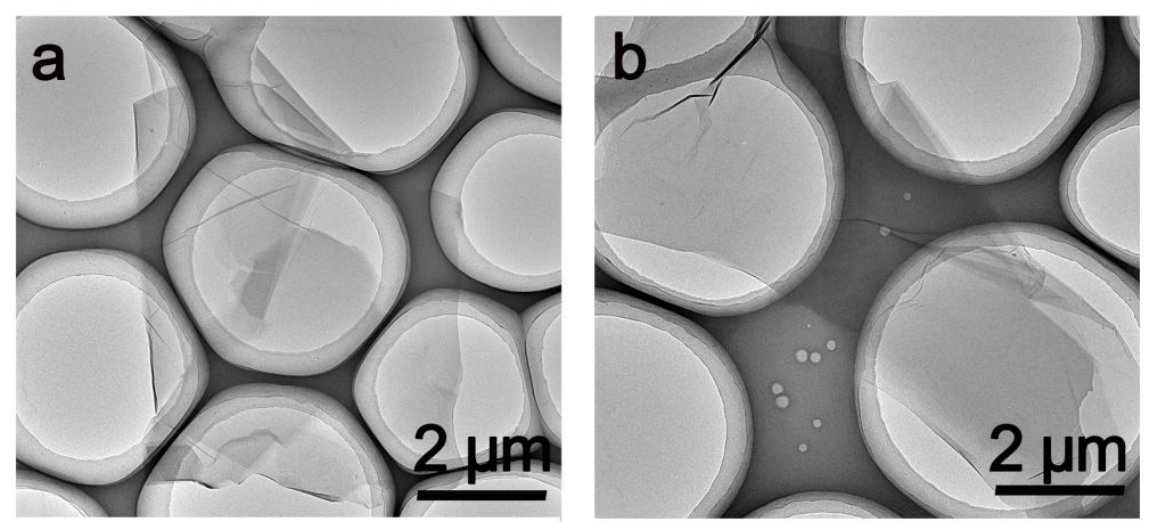

Figure S3. TEM images of (a) GO and (b) GO-ADP-Li $i_{3}$.
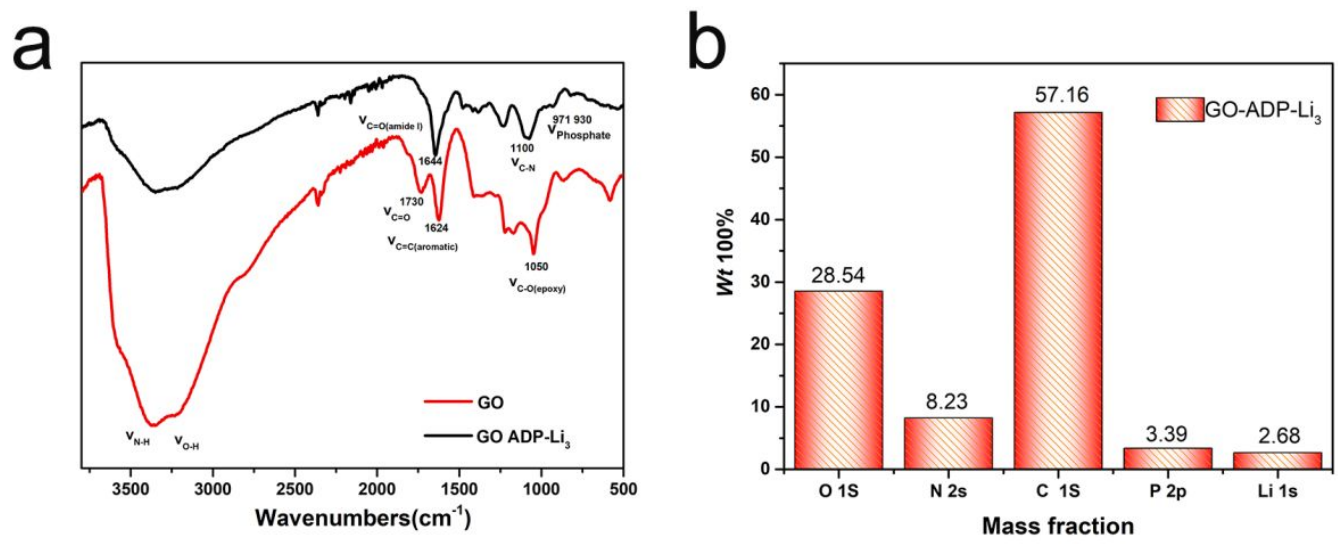

Figure S4. (a) FTIR spectra of GO-ADP-Li ${ }_{3}$ and GO. (b)The mass fraction of element $\mathrm{O}, \mathrm{N}, \mathrm{C}, \mathrm{P}$ and $\mathrm{Li}$ in GO-ADP- $\mathrm{Li}_{3}$ from the results of XPS. 

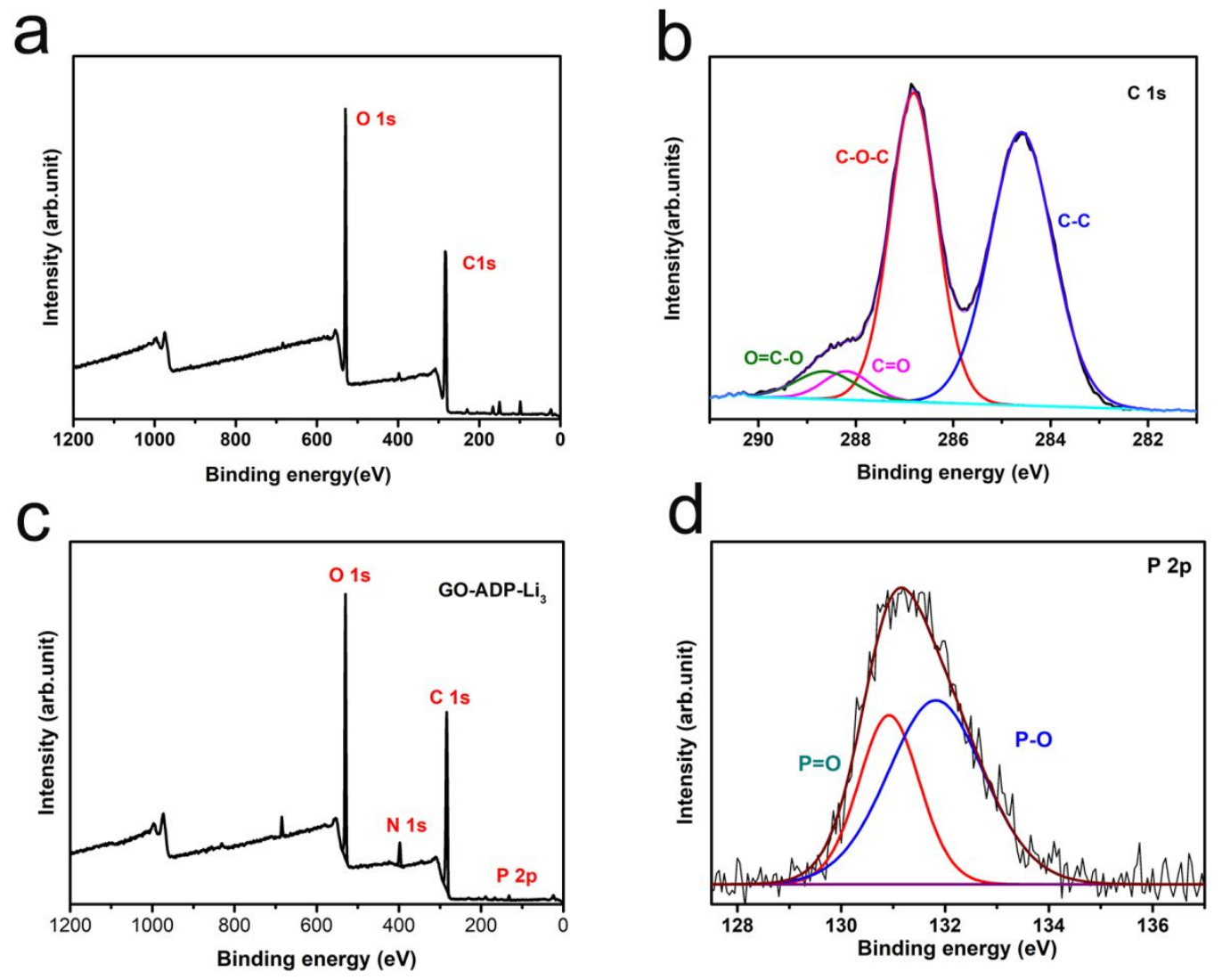

Figure S5. XPS spectra of (a) GO and (c) GO-ADP-Li $i_{3}$. (b) C 1s spectra of GO. (d) P $2 p$ spectra of GO-ADP- $\mathrm{Li}_{3}$.

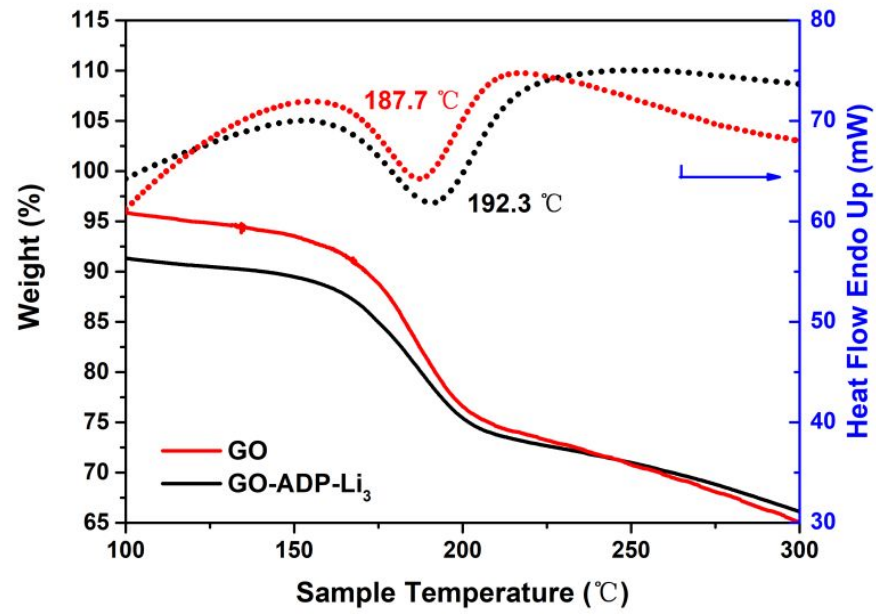

Figure S6. Thermogravimetric analysis curves (TGA) of GO-ADP-Li ${ }_{3}$ and GO. 

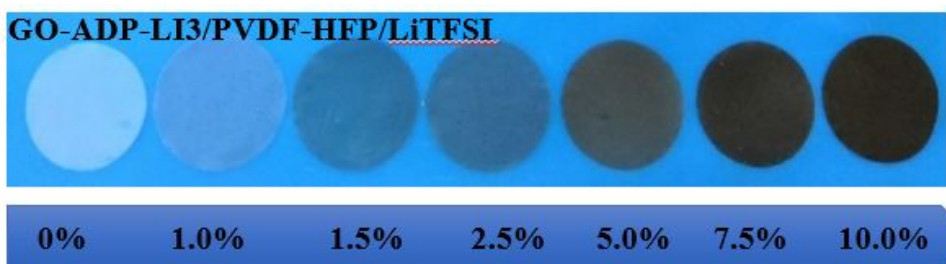

Figure S7. Digital photograph of solid electrolytes with $1.0 \mathrm{~g}$ PVDF-HFP, 0.5g LiTFSI and $\mathrm{x} w t . \%$ of GO-ADP-Li $3(\mathrm{x}=0,1.0,1.5,2.5,5.0,7.5$ and 10.0).

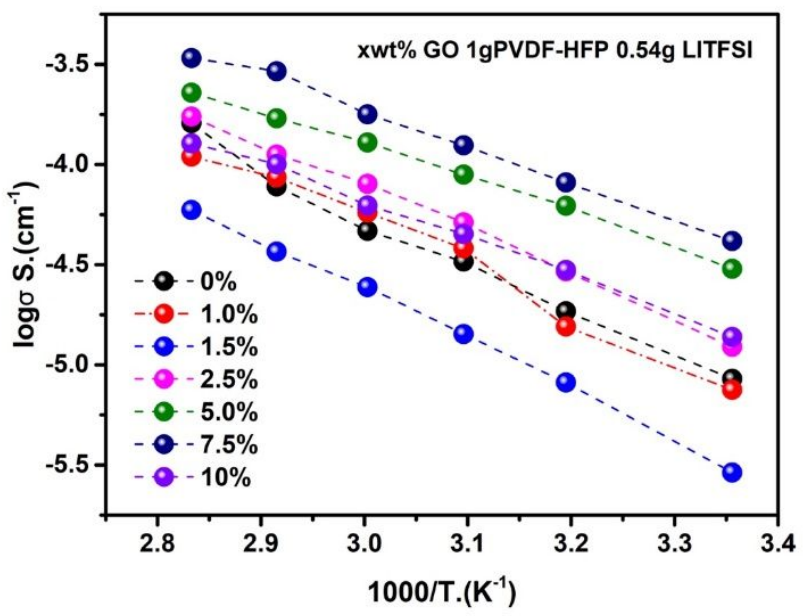

Figure S8. Arrhenius plots of ionic conductivity versus temperature for different mass fractions of GO in SPE. 

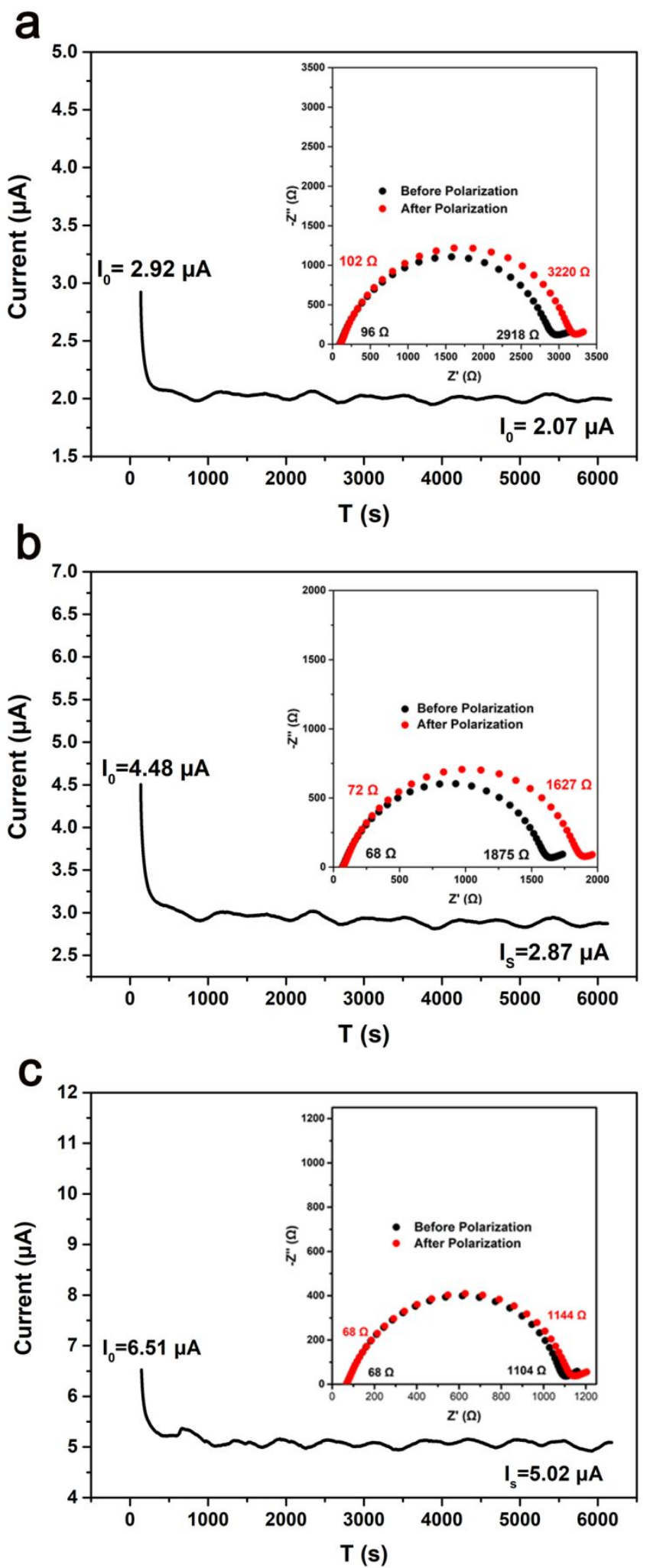

Figure S9. Li-ion transference numbers $\left(\mathrm{t}_{\mathrm{Li}}{ }^{+}\right)$of (a) SPE, (b) GO-SPE and (c) Li-GOSPE. 

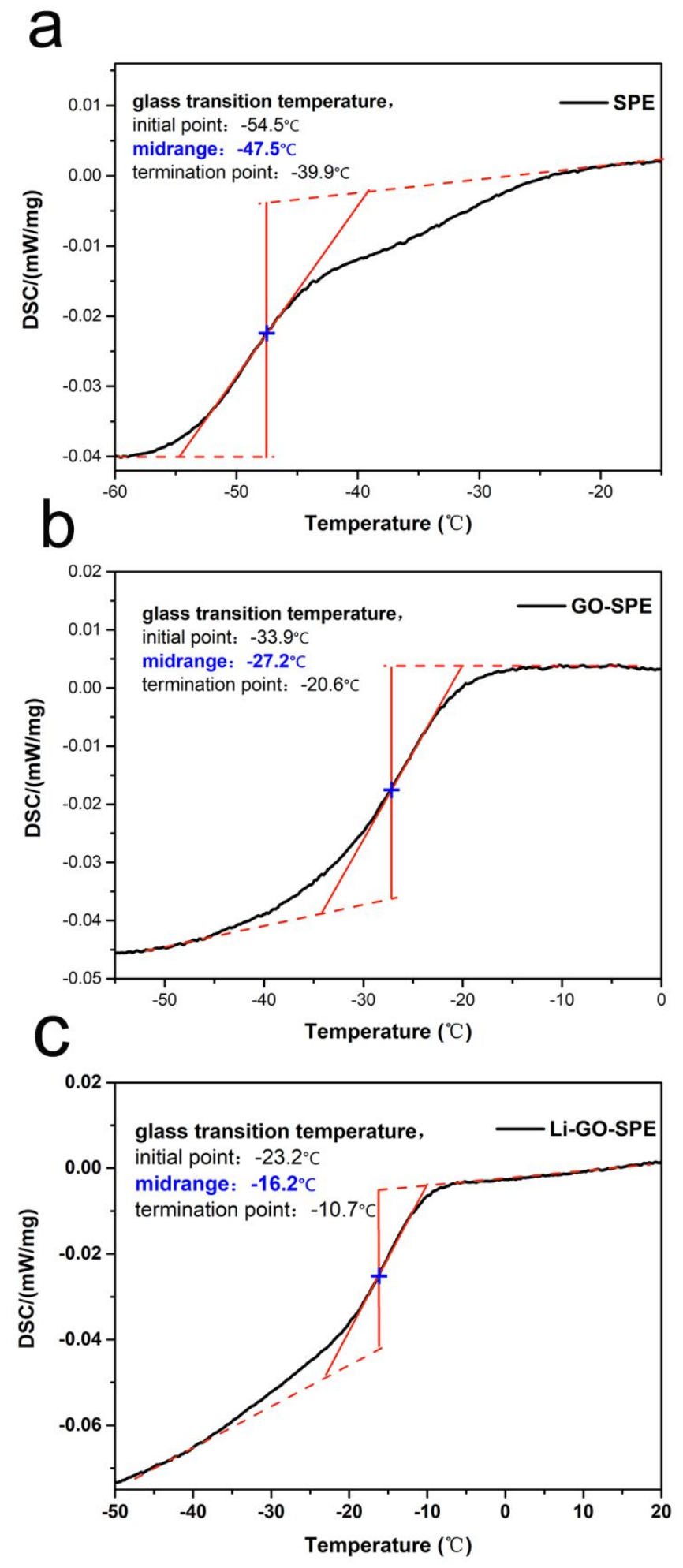

Figure S10. Differential scanning calorimetry curves of (a) SPE, (b) GO-SPE and (c) Li-GO-SPE for determination of the glass transition temperature. 


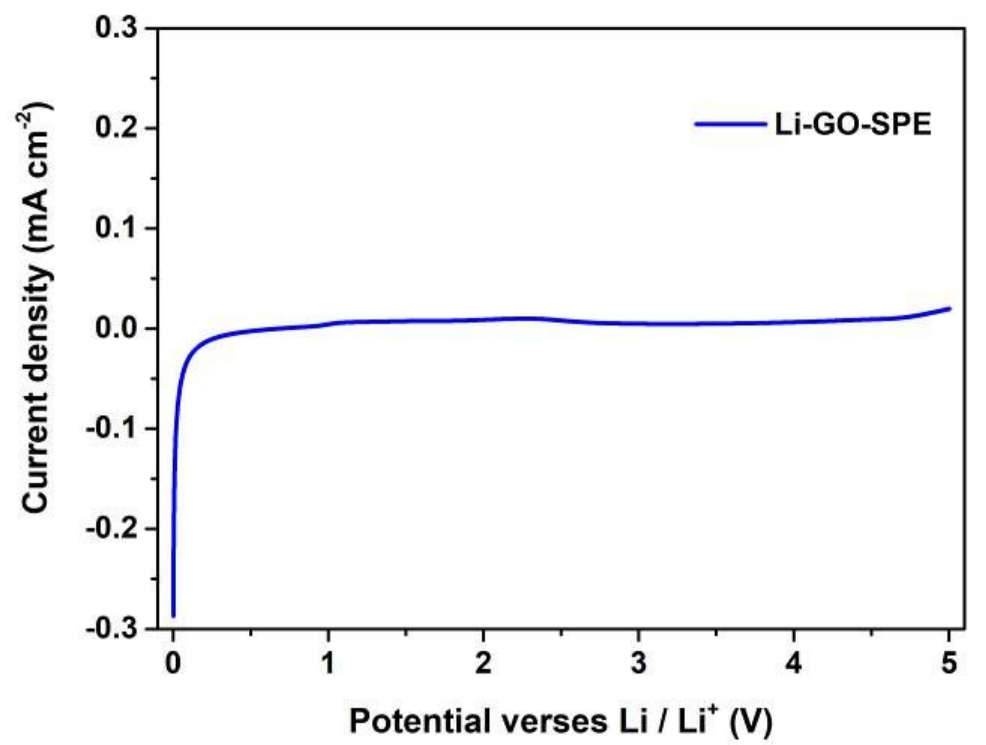

Figure S11. Linear sweep voltammogram (LSV) curves of Li-GO-SPE at room temperature with a scan rate of $0.5 \mathrm{mV} \mathrm{s}^{-1}$ and scan range of $0 \sim 5 \mathrm{~V}$.

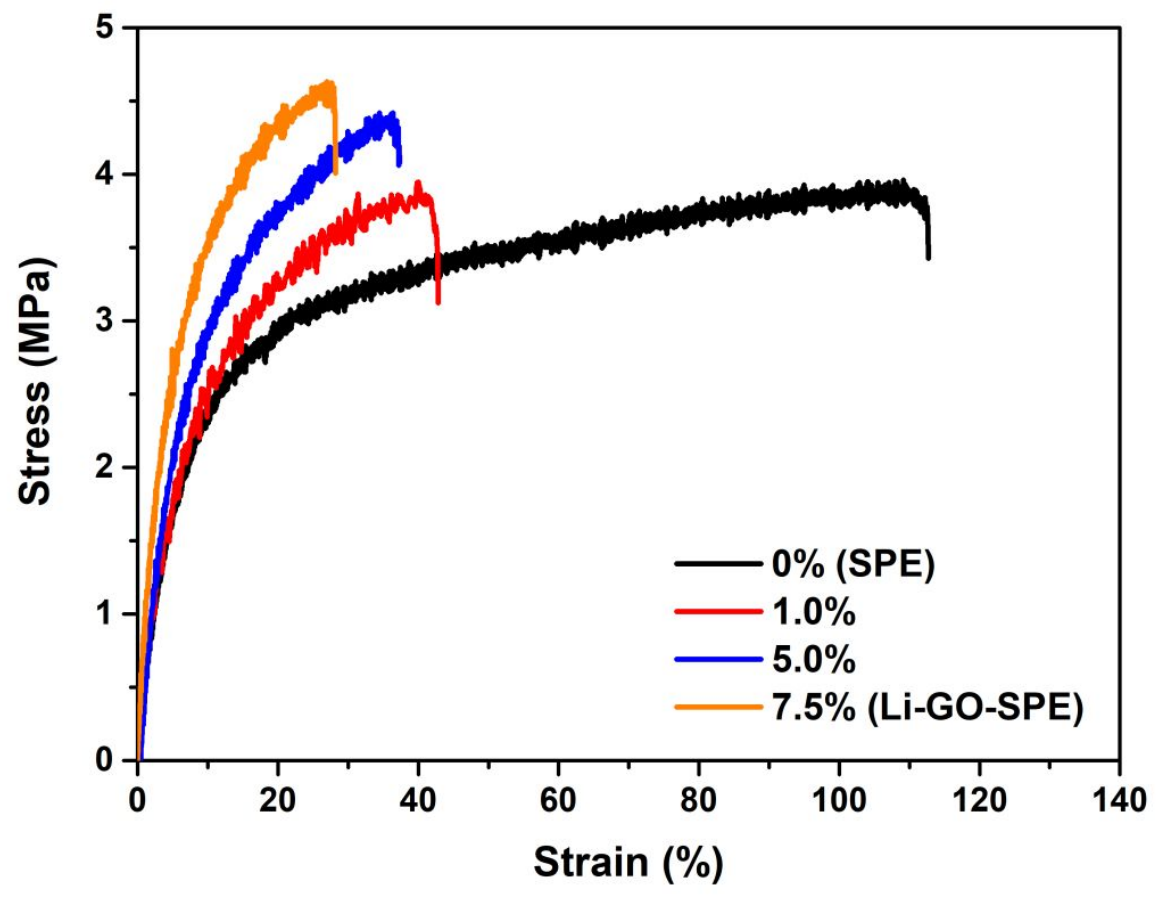

Figure S12. The mechanical property (stress-strain curves) of SPE composites with different weight fraction of GO-ADP-Li ${ }_{3}$ additives. 


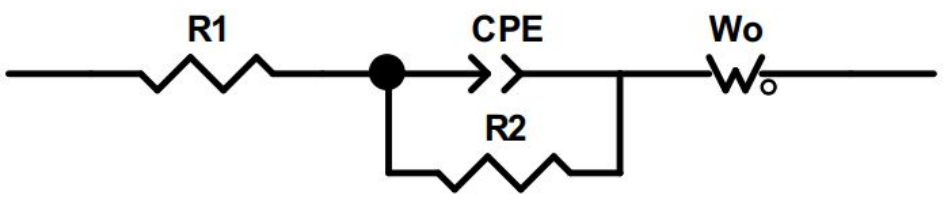

Figure $\mathrm{S} 13$. The equivalent circuit of bare $\mathrm{Cu}$ foil in $\mathrm{Li} / \mathrm{Cu}$ cell.

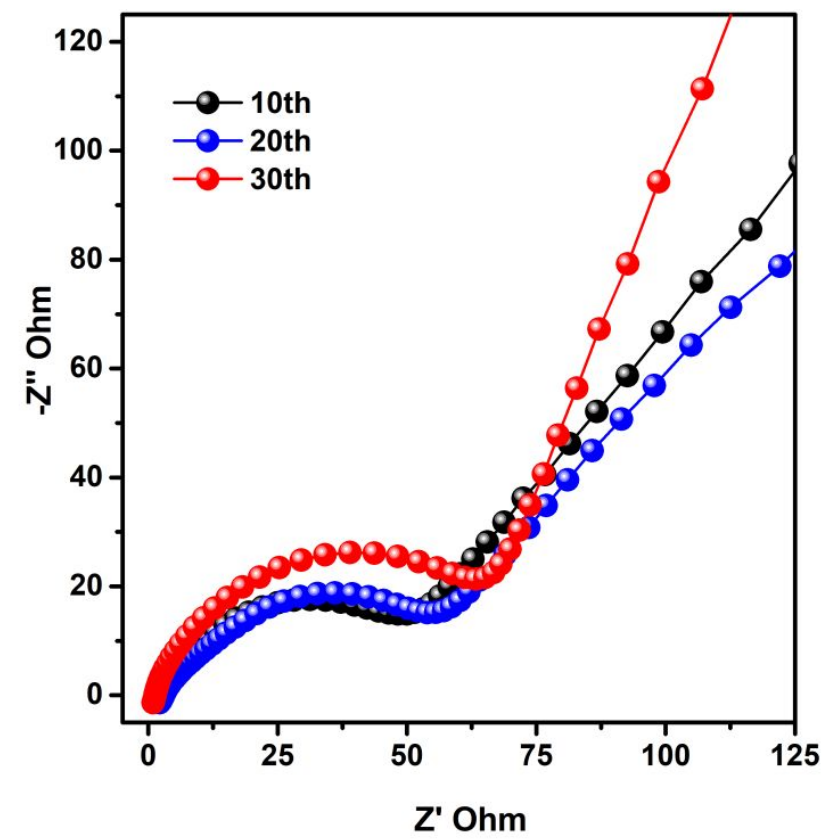

Figure S14. EIS profiles of GO-SPE@Cu foil at the $10^{\text {th }} / 20^{\text {th }} / 30^{\text {th }}$ cycle. 

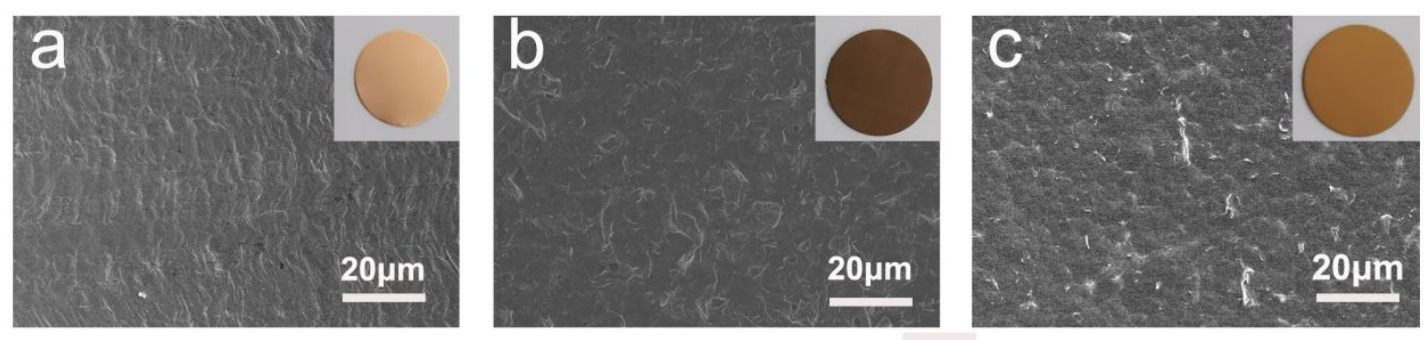

Figure S15. Top-view SEM images of (a) bare Cu, (b) GO-SPE@Cu foil and (c) LiGO-SPE@Cu foil.
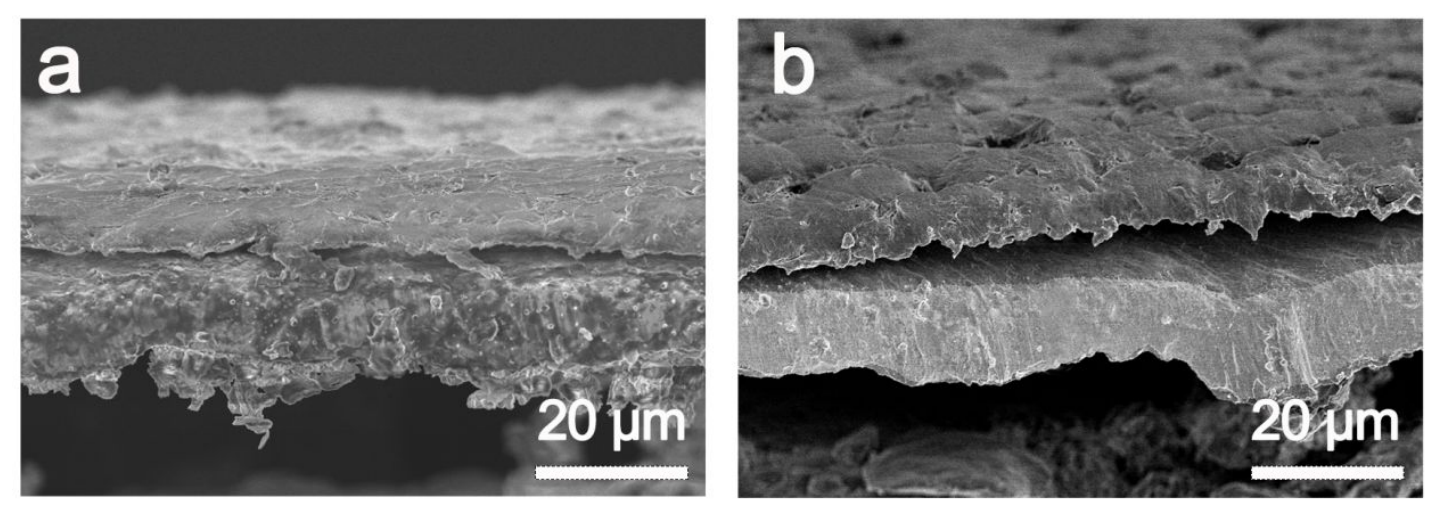

Figure S16. Cross-sectional SEM image of (a) GO-SPE@Cu foil and (b) Li-GOSPE@Cu foil. 


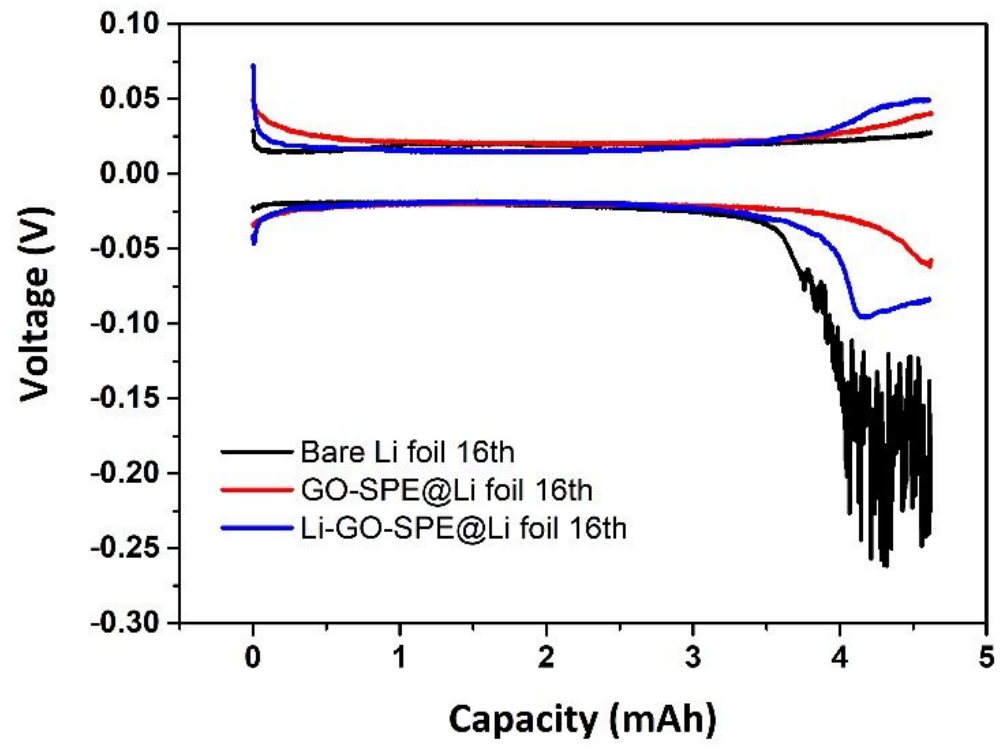

Figure S17. Capacity-voltage curves of bare Li foil, GO-SPE@Li foil and Li-GOSPE@Li foil in Li symmetrical cell at the $16^{\text {th }}$ cycle.
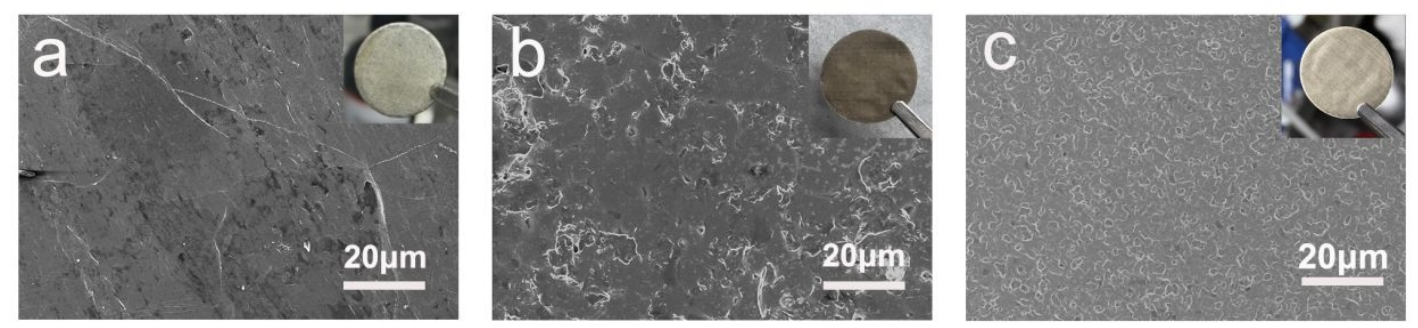

Figure S18. Top-view SEM images of (a) bare Li foil, (b) GO-SPE@Li foil and (c) LiGO-SPE@Li foil. 

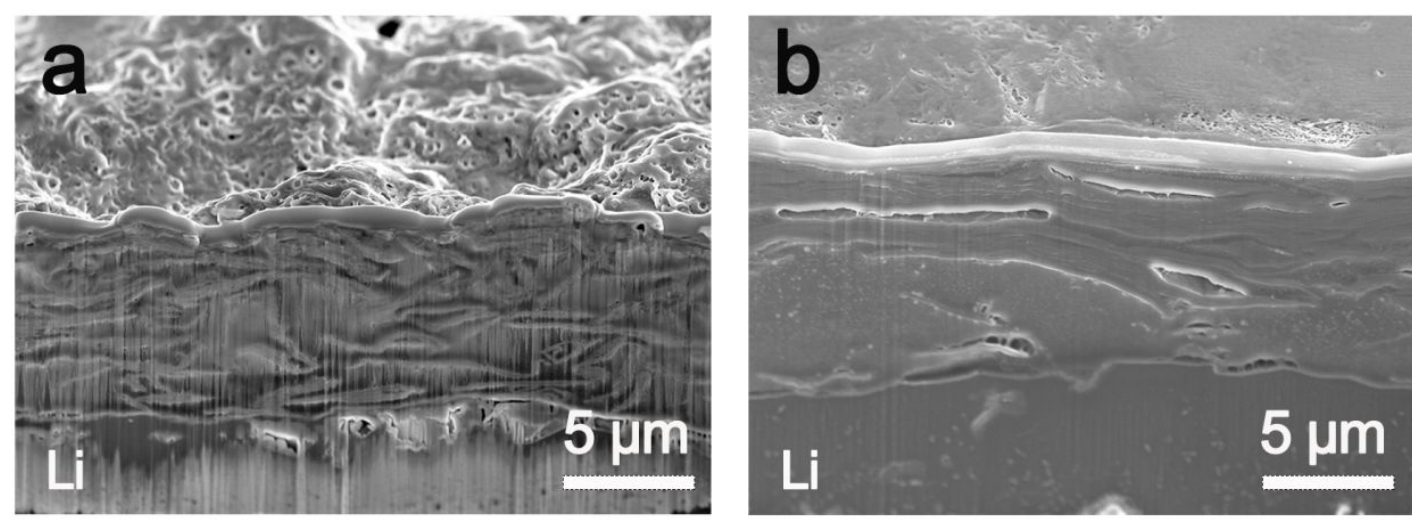

Figure S19. FIB-SEM image of (a) GO-SPE@Li foil and (b) Li-GO-SPE@Li foil.
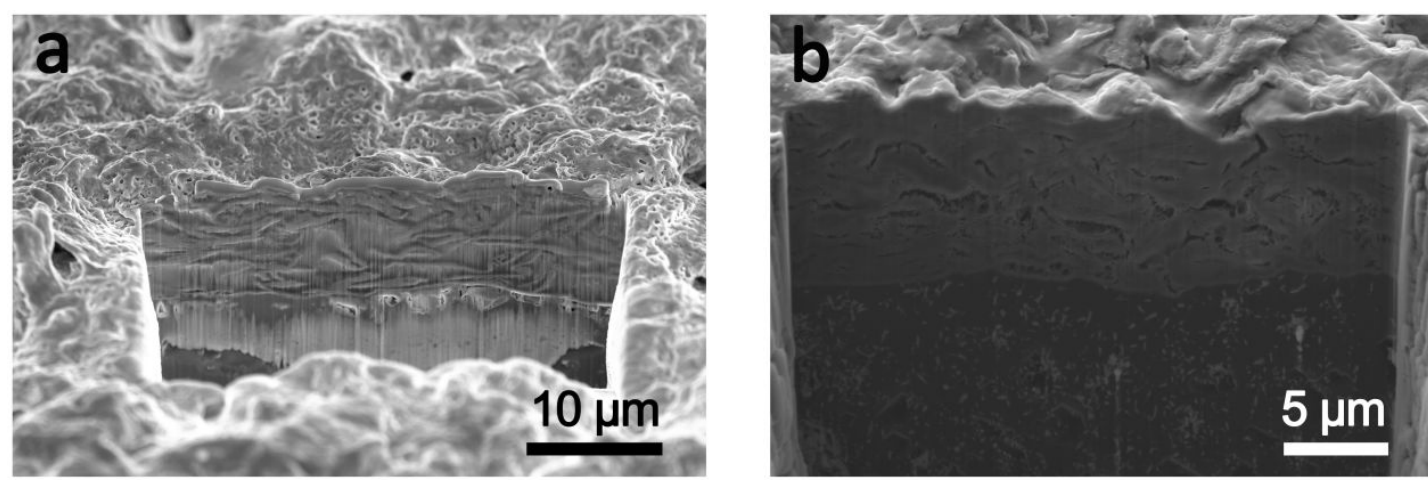

Figure S20. FIB-SEM image of GO-SPE@Li foil before cycling (a) and after 5 cycles

(b) under the same condition in Figure 4a. 


\section{Supporting Tables}

Table S1. Ionic conductivities and other parameters derived from the VTF fit.

\begin{tabular}{|c|c|c|c|c|c|c|}
\hline \multirow{2}{*}{ Electrolyte } & \multicolumn{3}{|c|}{$\sigma\left(\mathrm{S} \mathrm{cm}^{-1}\right)$} & \multirow{2}{*}{$\begin{array}{c}\ln \mathrm{A} \\
\left(\mathrm{S} \mathrm{cm}^{-1}\right. \\
\left.\mathrm{K}^{-1 / 2}\right)\end{array}$} & \multirow{2}{*}{$\begin{array}{c}\mathrm{Ea} \\
(\mathrm{KJ} \\
\left.\mathrm{mol}^{-1}\right)\end{array}$} & \multirow{2}{*}{$\begin{array}{c}\mathrm{T}_{0} \approx \mathrm{Tg} \\
(\mathrm{K})\end{array}$} \\
\hline & $40^{\circ} \mathrm{C}$ & $60^{\circ} \mathrm{C}$ & $80^{\circ} \mathrm{C}$ & & & \\
\hline SPE & $1.08 \times 10^{-5}$ & $\begin{array}{c}2.81 \times 10^{-} \\
5\end{array}$ & $\begin{array}{c}6.21 \times 10^{-} \\
5\end{array}$ & 5.31 & 12.787 & 256.95 \\
\hline GO-SPE & $8.21 \times 10^{-5}$ & $\begin{array}{c}1.78 \times 10^{-} \\
4\end{array}$ & $\begin{array}{c}3.39 \times 10^{-} \\
4\end{array}$ & 3.56 & 7.679 & 245.90 \\
\hline Li-GO-SPE & $1.64 \times 10^{-4}$ & $\begin{array}{c}3.33 \times 10^{-} \\
4\end{array}$ & $\begin{array}{c}7.59 \times 10^{-} \\
4\end{array}$ & 2.35 & 5.278 & 225.65 \\
\hline
\end{tabular}

Table S2. Li-ion transference numbers $\left(\mathrm{t}_{\mathrm{Li}}{ }^{+}\right)$of SPE, GO-SPE and Li-GO-SPE at room temperature.

\begin{tabular}{cccccccc}
\hline Electrolyte & $I_{0}(\mu \mathrm{A})$ & $I_{\mathrm{S}}(\mu \mathrm{A})$ & $R_{\mathrm{b} 0}(\Omega)$ & $R_{\mathrm{bS}}(\Omega)$ & $R_{0}(\Omega)$ & $R_{\mathrm{S}}(\Omega)$ & $t_{\mathrm{Li}}{ }^{+}$ \\
\hline SPE & 2.92 & 2.07 & 96 & 102 & 2918 & 3220 & 0.33 \\
GO-SPE & 4.48 & 2.87 & 68 & 72 & 1627 & 1875 & 0.40 \\
Li-GO-SPE & 6.51 & 5.02 & 68 & 68 & 1104 & 1144 & 0.51 \\
\hline
\end{tabular}


Table S3. The comparison on vital parameters of symmetric Li cells in this work with previously reported works.

\begin{tabular}{|c|c|c|c|c|c|c|c|}
\hline & \multicolumn{4}{|c|}{ symmetric Li cells } & \multirow[b]{2}{*}{$\mathrm{t}_{\mathrm{Li}}{ }^{+}$} & \multirow{2}{*}{$\begin{array}{c}\text { Li ionic } \\
\text { Conductivity } \\
/ \text { Temperture } \\
\left(\mathrm{S} \mathrm{cm}^{\left.-1 /{ }^{\circ} \mathrm{C}\right)}\right.\end{array}$} & \multirow[b]{2}{*}{ Reference ID } \\
\hline & Lithium Anode & $\begin{array}{c}\text { Li Anode } \\
\text { Thickness } \\
(\mu \mathrm{m})\end{array}$ & $\begin{array}{c}\text { Current Density/ } \\
\text { Capacity } \\
\left(\mathrm{mAcm}^{-2} / \mathrm{mAhcm}^{-2}\right)\end{array}$ & $\begin{array}{l}\text { Time } \\
\text { (h) }\end{array}$ & & & \\
\hline This work & Li-GO-SPE @Li foil & 100 & $0.5--3$ & 700 & 0.51 & $8.28 \times 10^{-5} \quad 25^{\circ} \mathrm{C}$ & \\
\hline Ref. $1^{3}$ & $\begin{array}{c}\text { PVDF-LiFSI } \\
\text { solid electrolyte }\end{array}$ & 600 & $0.05--0.05$ & 2000 & - & $1.18 \times 10^{-4}$ & Adv.Mater.2019, 31, 1806082 \\
\hline Ref. $2^{4}$ & $\begin{array}{l}\text { PEOm-5\% } 5 \mathrm{Li}_{2} 1 \mathrm{Si}_{5} \text { solid } \\
\text { electrolyte }\end{array}$ & - & $0.2--0.1$ & 300 & 0.43 & $\begin{array}{c}3.9^{*} 10^{-5} \\
30^{\circ} \mathrm{C}\end{array}$ & Adv.Mater.2021, 33,2004711 \\
\hline Ref. $3^{5}$ & $\begin{array}{c}\text { PVDF-HFP/LiF Artificial } \\
\text { Protective Layer }\end{array}$ & - & $2--1$ & 200 & - & - & $\begin{array}{l}\text { Advanced Functional Materials } \\
\qquad 2018,28(8)\end{array}$ \\
\hline Ref. $4^{6}$ & PI/PEO/LiTFSI SPE & 600 & $0.1--0.1$ & 1000 & - & $\begin{array}{c}2.3 \times 10^{-4} \\
30^{\circ} \mathrm{C} \\
\end{array}$ & $\begin{array}{c}\text { Nat Nanotechnol 2019, } 14 \text { (7), } \\
705-711 \\
\end{array}$ \\
\hline Ref. $5^{7}$ & $\mathrm{Mg} @ \mathrm{C}_{60} @ \mathrm{Li}$ & 600 & $1--1$ & 550 & - & - & $\begin{array}{l}\text { Advanced Energy Materials } \\
2019,10(6)\end{array}$ \\
\hline Ref. $6^{8}$ & $\begin{array}{l}\text { Polymer-Reinforced SEI } \\
\text { Layers }\end{array}$ & 600 & $0.5--0.5$ & 1000 & 0.44 & $\begin{array}{c}1.27 \times 10^{-3} \mathrm{~S} \\
30^{\circ} \mathrm{C}\end{array}$ & Small 2020, 16 (13), e1907163 \\
\hline Ref. $7^{9}$ & $\begin{array}{c}\text { Double-Layered Polymer } \\
\text { Electrolyte(DLPEs) }\end{array}$ & 600 & $0.05--0.1$ & 1000 & 0.48 & $\begin{array}{c}1.56 \times 10^{-4} \\
20^{\circ} \mathrm{C}\end{array}$ & $\begin{array}{l}\text { Energy Storage Materials 2020, } \\
32,55-64\end{array}$ \\
\hline $\operatorname{Ref} .8^{10}$ & $\begin{array}{l}\mathrm{LiNO}_{3} \text {-derived multilayer- } \\
\text { structured SEI (N-SEI) }\end{array}$ & - & $1--1$ & 800 & - & - & $\begin{array}{c}\text { Energy \& Environmental } \\
\text { Science 2021, } 14 \text { (3), 1439-1448 }\end{array}$ \\
\hline
\end{tabular}


Table S4. The comparison on vital parameters and cycle life of Li metal full cells in this work with previously reported works.

\begin{tabular}{|c|c|c|c|c|c|c|c|c|c|}
\hline & Anode & Cathode & $\begin{array}{c}\text { Rate } \\
\text { C }\end{array}$ & $\begin{array}{c}\text { Capacity } \\
\text { (Ah) }\end{array}$ & $\begin{array}{l}\text { Electrolyte } \\
\text { Injection }\end{array}$ & $\mathrm{N} / \mathrm{P}$ & $\begin{array}{l}\text { Cycle life } \\
\text { (cycle number- } \\
\text { retention) }\end{array}$ & $\begin{array}{l}\text { Cell } \\
\text { type }\end{array}$ & Reference ID \\
\hline $\begin{array}{r}\text { This } \\
\text { work }\end{array}$ & Li-GO-SPE@Li foil & NCM811 & 0.2 & 0.2 & $3 \mathrm{~g} / \mathrm{Ah}$ & 2.66 & $150-78.4 \%$ & $\begin{array}{c}\text { Pouch } \\
\text { cell }\end{array}$ & \\
\hline Ref. $1^{11}$ & $\mathrm{Li}-\mathrm{Sb}-\mathrm{Sn}$ & NCM811 & 0.3 & - & $13.33 \mathrm{~g} / \mathrm{Ah}$ & - & $100--85.4 \%$ & $\begin{array}{c}\text { Coin } \\
\text { cell }\end{array}$ & $\begin{array}{c}\text { Nano Energy } \\
74(2020) 104815\end{array}$ \\
\hline Ref. $2^{12}$ & $\mathrm{Li}$ & NCM622 & 0.1 & 1 & $3 \mathrm{~g} / \mathrm{Ah}$ & 2.6 & $200--86 \%$ & $\begin{array}{c}\text { Pouch } \\
\text { cell }\end{array}$ & $\begin{array}{c}\text { Nature Energy } \\
2019,4(7), 551-559 \\
\end{array}$ \\
\hline Ref. $3^{13}$ & Graphite- $\mathrm{SiO}_{2} \mathrm{Li}$ & NMC111 & 0.1 & - & $60 \mu \mathrm{L}$ & - & $150--74.21 \%$ & $\begin{array}{c}\text { Coin } \\
\text { cell }\end{array}$ & $\begin{array}{c}\text { Adv. Energy Mater. } \\
2019,1901486 \\
\end{array}$ \\
\hline Ref. $4^{14}$ & Housed Li & $\mathrm{LiFePO}_{4}$ & 0.5 & 0.45 & - & - & $80--85 \%$ & $\begin{array}{c}\text { Pouch } \\
\text { cell }\end{array}$ & $\begin{array}{c}\text { Journal of Energy } \\
\text { Chemistry } 2019,37,29-34\end{array}$ \\
\hline Ref. $5^{15}$ & $\begin{array}{c}\text { CN500@Li anode and } \\
\text { A-ELY }\end{array}$ & NCA & 0.1 & - & $4 \mathrm{~g} / \mathrm{Ah}$ & 2 & $100--76.5 \%$ & $\begin{array}{c}\text { Coin } \\
\text { cell }\end{array}$ & $\begin{array}{c}\text { Energy Storage Materials } \\
2021,37,491-500\end{array}$ \\
\hline Ref. $6^{16}$ & LiPON@Li & $\mathrm{LiCoO}_{2}$ & 0.1 & 0.14 & $13.7 \mathrm{~g} / \mathrm{Ah}$ & 29.87 & $100--80.67 \%$ & $\begin{array}{l}\text { Pouch } \\
\text { cell }\end{array}$ & $\begin{array}{c}\text { ACS Applied Energy } \\
\text { Materials 2018, } 1 \text { (4), } \\
1674-1679\end{array}$ \\
\hline
\end{tabular}




\section{REFERENCES}

(1) Deng, W.; Zhou, X.; Fang, Q.; Liu, Z. Microscale lithium metal stored inside cellular graphene scaffold toward advanced metallic lithium anodes. Adv. Energy Mater. 2018, $8(12), 1703152$.

(2) Deng, W.; Dai, W.; Zhou, X.; Han, Q.; Fang, W.; Dong, N.; He, B.; Liu, Z. Competitive Solvation-Induced Concurrent Protection on the Anode and Cathode toward a $400 \mathrm{Wh}$ kg-1 Lithium Metal Battery. ACS Energy Lett. 2020, 115-123.

(3) Zhang, X.; Wang, S.; Xue, C.; Xin, C.; Lin, Y.; Shen, Y.; Li, L.; Nan, C. W. SelfSuppression of Lithium Dendrite in All-Solid-State Lithium Metal Batteries with Poly(vinylidene difluoride)-Based Solid Electrolytes. Adv Mater 2019, 31 (11), 1806082.

(4) Liu, Y.; Hu, R.; Zhang, D.; Liu, J.; Liu, F.; Cui, J.; Lin, Z.; Wu, J.; Zhu, M. Constructing Li-Rich Artificial SEI Layer in Alloy-Polymer Composite Electrolyte to Achieve High Ionic Conductivity for All-Solid-State Lithium Metal Batteries. Adv Mater 2021, 33 (11), 2004711.

(5) Xu, R.; Zhang, X.-Q.; Cheng, X.-B.; Peng, H.-J.; Zhao, C.-Z.; Yan, C.; Huang, J.-

Q. Artificial Soft-Rigid Protective Layer for Dendrite-Free Lithium Metal Anode. Advanced Functional Materials 2018, 28 (8), 1705838.

(6) Wan, J.; Xie, J.; Kong, X.; Liu, Z.; Liu, K.; Shi, F.; Pei, A.; Chen, H.; Chen, W.; Chen, J.; Zhang, X.; Zong, L.; Wang, J.; Chen, L. Q.; Qin, J.; Cui, Y. Ultrathin, flexible, solid polymer composite electrolyte enabled with aligned nanoporous host for lithium batteries. Nat Nanotechnol 2019, 14 (7), 705-711. 
(7) Xu, Q.; Lin, J.; Ye, C.; Jin, X.; Ye, D.; Lu, Y.; Zhou, G.; Qiu, Y.; Li, W. Air-Stable and Dendrite-Free Lithium Metal Anodes Enabled by a Hybrid Interphase of $\mathrm{C}_{60}$ and Mg. Advanced Energy Materials 2019, 10 (6), 1903292.

(8) Hu, R.; Qiu, H.; Zhang, H.; Wang, P.; Du, X.; Ma, J.; Wu, T.; Lu, C.; Zhou, X.; Cui, G. A Polymer-Reinforced SEI Layer Induced by a Cyclic Carbonate-Based Polymer Electrolyte Boosting 4.45 V LiCoO 2 /Li Metal Batteries. Small 2020, 16 (13), 1907163.

(9) Wen, K.; Tan, X.; Chen, T.; Chen, S.; Zhang, S. Fast Li-ion transport and uniform Li-ion flux enabled by a double-layered polymer electrolyte for high performance $\mathrm{Li}$ metal battery. Energy Storage Materials 2020, 32, 55-64.

(10) Lin, X.-D.; Gu, Y.; Shen, X.-R.; Wang, W.-W.; Hong, Y.-H.; Wu, Q.-H.; Zhou, Z.-Y.; Wu, D.-Y.; Chang, J.-K.; Zheng, M.-S.; Mao, B.-W.; Dong, Q.-F. An oxygenblocking oriented multifunctional solid-electrolyte interphase as a protective layer for a lithium metal anode in lithium-oxygen batteries. Energy \& Environmental Science 2021, 14 (3), 1439-1448.

(11) Xu, H.; Li, S.; Chen, X.; Zhang, C.; Tang, Z.; Fan, H.; Yu, Y.; Liu, W.; Liang, N.; Huang, Y.; Li, J. Surpassing lithium metal rechargeable batteries with self-supporting Li-Sn-Sb foil anode. Nano Energy 2020, 74, 104815.

(12) Niu, C.; Lee, H.; Chen, S.; Li, Q.; Du, J.; Xu, W.; Zhang, J.-G.; Whittingham, M. S.; Xiao, J.; Liu, J. High-energy lithium metal pouch cells with limited anode swelling and long stable cycles. Nature Energy 2019, 4 (7), 551-559.

(13) Pathak, R.; Chen, K.; Gurung, A.; Reza, K. M.; Bahrami, B.; Wu, F.; Chaudhary, A.; Ghimire, N.; Zhou, B.; Zhang, W. H.; Zhou, Y.; Qiao, Q. Ultrathin Bilayer of 
Graphite/ $\mathrm{SiO}_{2}$ as Solid Interface for Reviving Li Metal Anode. Advanced Energy Materials 2019, 9 (36), 1901486.

(14) Shen, X.; Cheng, X.; Shi, P.; Huang, J.; Zhang, X.; Yan, C.; Li, T.; Zhang, Q. Lithium-matrix composite anode protected by a solid electrolyte layer for stable lithium metal batteries. Journal of Energy Chemistry 2019, 37, 29-34.

(15) Li, Z.; Huai, L.; Li, S.; Ma, M.; Luo, K.; Zhao, Y.; Wang, D.; Sun, X.; Peng, Z. Insight into bulk charge transfer of lithium metal anodes by synergism of nickel seeding and $\mathrm{LiF}_{-} \mathrm{Li}_{3} \mathrm{~N}-\mathrm{Li}_{2} \mathrm{~S}$ co-doped interphase. Energy Storage Materials 2021, 37, 491-500. (16) Liu, W.; Guo, R.; Zhan, B.; Shi, B.; Li, Y.; Pei, H.; Wang, Y.; Shi, W.; Fu, Z.; Xie, J. Artificial Solid Electrolyte Interphase Layer for Lithium Metal Anode in HighEnergy Lithium Secondary Pouch Cells. ACS Applied Energy Materials 2018, 1 (4), 1674-1679. 